\title{
Percepção sobre prevenção primária do câncer de mama entre mulheres atendidas no Sistema Único de Saúde
}

Current awareness on primary prevention of breast cancer among Unified Health System insured

brazilian women

Percepción sobre prevención primaria del cáncer de mama en mujeres atendidas en el Sistema Único de Salud

Renata Capanema Saliba Franco ORCID: https://orcid.org/0000-0003-3127-1869 Rede Mater Dei, Brasil E-mail: saliba.renata@gmail.com

Fernanda Cardoso Parreiras ORCID: https://orcid.org/0000-0002-5177-8600 Universidade José do Rosário Vellano, Brasil E-mail: fernanda.med@me.com

Sávia Vieira Rosembarque ORCID: https://orcid.org/0000-0002-5362-2298 Universidade José do Rosário Vellano, Brasil E-mail: saviarosembarque@gmail.com

Gabriela Belizário Lasmar

ORCID: https://orcid.org/0000-0003-2082-4042 Universidade José do Rosário Vellano, Brasil E-mail: gabiblasmar@gmail.com Heloisa Malaquias Vidal ORCID: https://orcid.org/0000-0002-4278-761X Universidade José do Rosário Vellano, Brasil E-mail: lohvidal@gmail.com

Nathalia Ornelas Ribeiro Chaves ORCID: https://orcid.org/0000-0002-6897-7294 Universidade José do Rosário Vellano, Brasil E-mail: nathy_orc@hotmail.com

\begin{abstract}
Resumo
O câncer de mama configura-se como importante problema de saúde pública. A prevenção primária busca reduzir a exposição aos fatores de risco e estimula a mudança de estilo de vida. O presente estudo tem como objetivo avaliar o conhecimento e as fontes de informações sobre os fatores de risco para câncer de mama entre mulheres de um centro de atenção secundária da região Sudeste do Brasil, na cidade de Belo Horizonte. Trata-se de um estudo transversal, cuja coleta de dados foi realizada por meio de um questionário semiestruturado que incluiu variáveis demográficas (como idade, escolaridade, estado civil e ocupação) e a pesquisa acerca de fontes de informação, acesso à informação, fatores de risco e causas do câncer de mama. Foram incluídas 398 mulheres, com faixa etária entre 35 e 69 anos. A fonte de informação mais apontada foi a mídia, seguida da Unidade Básica de Saúde e 79,6\% das mulheres nunca participaram de atividades do outubro rosa. Foram listados 12 itens como possíveis fatores de risco, sendo sete modificáveis. A média de acertos foi de apenas 1,38 e 1,76, respectivamente. O fator de risco mais reconhecido foi a história familiar de câncer de mama e a "genética" foi descrita como a principal causa da doença. Os dados evidenciam o limitado conhecimento, bem como o reduzido alcance das ações de saúde pública voltadas para a promoção da saúde, o que demonstra a necessidade de reformulação para a que as informações cheguem ao públicoalvo. Ademais, é essencial que as mulheres saibam da sua responsabilidade individual, adotando um estilo de vida saudável.
\end{abstract}

Palavras-chave: Neoplasia de mama; Prevenção primária; Promoção da saúde.

\section{Abstract}

Breast cancer is an important public health problem. Primary prevention seeks to reduce exposure to risk factors and encourages lifestyle changes. This study aims to assess knowledge and sources of information about risk factors for breast cancer among women at a secondary care center in Belo Horizonte, Brazil. This is a cross-sectional study, whose data collection was performed using a semi-structured questionnaire that included demographic variables (such as age, education, marital status and occupation) and research on information sources, access to information, risk 
factors and causes of breast cancer. A total of 398 women were included, aged between 35 and 69 years. The main source of information about Breast Cancer was the media, followed by the primary care unit. $79.6 \%$ of women have never participated in Pink October activities. Twelve items were listed as possible risk factors for breast cancer, and seven of those were modifiable risk factors. The average number of correct items identified was 1.38 and 1.76 , respectively. The most recognized risk factor was family history of breast cancer. Genetics was described as the main cause of the disease. Our data show the limited awareness, as well as the reduced scope of public health actions towards breast cancer health promotion. It demonstrates the urge for reformulation of the breast cancer awareness information until it reaches the target audience. In addition, it is essential that women assume their individual health responsibility, adopting a better lifestyle.

Keywords: Breast Neoplasms; Primary prevention; Health Promotion.

\section{Resumen}

El cáncer de mama es un importante problema de salud pública. La prevención primaria busca reducir la exposición a factores de riesgo y fomenta cambios en el estilo de vida. Este estudio tiene como objetivo evaluar el conocimiento y las fuentes de información sobre los factores de riesgo del cáncer de mama en mujeres que asisten a una unidad secundaria de salud en la región sureste de Brasil, en Belo Horizonte. Se trata de un estudio transversal, cuya recogida de datos se realizó mediante un cuestionario semiestructurado que incluyó variables demográficas (como edad, escolaridad, estado civil y ocupación) e investigación sobre fuentes de información, acceso a la información, factores de riesgo y causas de cáncer de mama. Se incluyó a un total de 398 mujeres, con edades comprendidas entre 35 y 69 años. La fuente de información más señalada fueron los medios de comunicación, seguidos de la Unidad Básica de Salud y el 79,6\% de las mujeres nunca participó en las actividades de Octubre Rosa. Se enumeraron 12 ítems como posibles factores de riesgo, 7 de los cuales pueden modificarse. El número medio de respuestas correctas fue de solo 1,38 y 1,76, respectivamente. El factor de riesgo más reconocido fueron los antecedentes familiares de cáncer de mama y se describió la "genética" como la principal causa de la enfermedad. Los datos evidencian el escaso conocimiento, así como el reducido alcance de las acciones de salud pública orientadas a la promoción de la salud, lo que demuestra la necesidad de reformulación para que la información llegue al público objetivo. Además, es fundamental que las mujeres conozcan su responsabilidad individual, adoptando un estilo de vida saludable.

Palabras clave: Cáncer de mama; Prevención primaria; Promoción de la salud.

\section{Introdução}

O câncer (CA) de mama é um tumor maligno que se desenvolve no tecido mamário. É a neoplasia de maior incidência na população feminina, excetuando-se os casos de câncer de pele não melanoma, e é rara em homens, representando apenas 1\% dos casos (Santos, 2018). As estimativas mostraram que em 2018 ocorreram 2,1 milhões de casos novos dessa doença no mundo, o que equivale a 11,6\% do total de cânceres estimados. As previsões para o Brasil são de que a cada ano, entre 2020 e 2022, ocorrerão 625 mil novos casos de câncer, sendo o de pele não melanoma o mais incidente, seguido das neoplasias da mama e da próstata, com 66 mil casos novos, segundo o Instituto Nacional de Câncer José Alencar Gomes da Silva (INCA, 2019). Tais dados corroboram a percepção do CA de mama como importante problema de saúde pública.

Empresas, famílias e a sociedade como um todo são afetadas pelos prejuízos causados pelo diagnóstico de CA de mama (Silva et al 2020). Entre os custos, é possível mencionar perda de produtividade no trabalho, consequências emocionais, aumento das despesas de saúde, redução da expectativa de vida e mortalidade precoce (Rodrigues, Cruz \& Paixão, 2015). O fortalecimento das medidas de prevenção para a doença é essencial, pois além de atuar na redução da alta morbimortalidade, também apresenta maior custo benefício do que o tratamento.

A prevenção primária refere-se a toda e qualquer ação voltada para o controle da exposição aos fatores de risco. Objetiva reduzir a ocorrência da doença através da promoção da saúde e proteção específica, compreendendo medidas relacionadas aos hábitos de vida. Por outro lado, a prevenção secundária abrange o conjunto de ações que permitem o diagnóstico precoce da doença e o seu tratamento imediato, constituindo do autoexame, exame clínico e rastreamento imaginológico das mamas realizado através da mamografia (Kolak et al, 2017).

Diversas condições relacionam-se com um maior risco de desenvolver o CA de mama, sendo muitas delas passíveis de prevenção. Os fatores de risco incluem dieta, ingestão alcoólica, excesso de peso, sedentarismo após a menopausa, exposição à radiação ionizante (World Health Organization [WHO], 2020), além de fatores endócrinos e da história 
reprodutiva - primeira gravidez após os 30 anos, não amamentar, nuliparidade, uso de contraceptivos orais, terapia hormonal na pós-menopausa, história de menarca precoce (anterior aos 12 anos) e menopausa tardia (após os 55 anos) (American Cancer Society [ACS], 2020). A idade avançada, sobretudo a partir dos 50 anos, e a influência genética são apontados como os principais fatores não modificáveis (Pinho \& Coutinho, 2005).

A identificação dos fatores de risco e as ações de promoção à saúde voltadas para seu combate são a base da prevenção primária das neoplasias da mama. É estimado que mudanças no estilo de vida podem reduzir de 25 a $30 \%$ dos casos da doença. Além disso, os estudos também demonstram a efetividade de atuar em fatores isolados. A redução do peso de pelo menos $5 \%$ pode reduzir o risco de CA de mama de 25 a $40 \%$ e o consumo de $10 \mathrm{~g}$ de álcool por dia aumenta o risco em 4 a $12 \%$ (Harvie et al, 2015).

Sendo assim, o presente estudo tem como objetivo avaliar o conhecimento e as fontes de informações sobre os fatores de risco para câncer de mama entre mulheres de um centro de atenção secundária da região Sudeste do Brasil, na cidade de Belo Horizonte.

\section{Metodologia}

Trata-se de um estudo transversal, em que os dados foram coletados no ano de 2019. Foram incluídas mulheres, com idade entre 35 e 69 anos, atendidas nos ambulatórios de especialidades médicas do Centro de Ensino e Atenção à Saúde da Comunidade (CEASC). Este local é referência para atendimento da população da regional norte na cidade de Belo Horizonte. Os critérios de exclusão foram: as acompanhantes das pacientes, mulheres analfabetas ou com limitação cognitiva que comprometa a realização do questionário.

O instrumento de coleta foi um questionário semiestruturado sobre prevenção primária do câncer de mama. Este investigava variáveis sociodemográficas e clínicas (idade, peso, altura, escolaridade, estado civil e ocupação) e o acesso à informação, através de locais (Unidades Básicas de Saúde - UBS e consultório particular) e veículos (redes sociais, televisão, jornais, revistas, amigos e campanhas de Outubro Rosa). Também apresentou 12 possíveis fatores de risco (idade, alimentação, atividade física, obesidade, consumo de bebidas alcoólicas, menarca antes dos 12 anos, menopausa após 55 anos, história familiar de câncer de mama, ter filhos, amamentar, terapia hormonal e uso de anticoncepcional hormonal), dentre os quais as mulheres eram encorajadas a marcar as alternativas que consideravam fatores de risco para a doença. Também havia uma pergunta aberta sobre qual seria a principal causa do CA de mama.

A avaliação da composição corporal foi realizada por meio da Classificação de Índice de massa corporal (IMC), de acordo com a Organização Mundial da Saúde $(\mathrm{OMS})$, que considera o peso e a altura $\left(\mathrm{IMC}=\right.$ peso/altura ${ }^{2}$ ). Ademais, as participantes foram classificadas quanto ao grau de escolaridade, que considera o ensino fundamental $\left(1^{\circ}\right.$ ao $9^{\circ}$ ano), o ensino médio ( $1^{\circ}$ ao $3^{\circ}$ ano), a graduação e a pós-graduação.

O estudo foi aprovado pelo Comitê de Ética e Pesquisa Sistema CEP/CONEP (CAAE: 02652018.0.0000.5143). As pacientes consentiram em participar e após assinarem o termo de consentimento livre esclarecido (TCLE), responderam o questionário na sala de espera, sem interferência do entrevistador.

Foi realizado o cálculo amostral com margem de erro de 4,9\% para mais ou para menos, dentro de um intervalo de confiança (IC) de 95\% através da calculadora Sample Size, sendo então selecionadas 398 pacientes. A análise estatística foi realizada através do programa Statistical Package for the Social Science (SPSS) 17.0. As variáveis contínuas foram expressas como média e desvio padrão ou mediana e intervalo de confiança, enquanto as variáveis categóricas foram expressas como frequência absoluta e relativa. Foram apresentadas medidas descritivas e percentuais para descrever os resultados das variáveis de interesse. Alguns dados também foram analisados através de Regressão Logística Binária Univariada. Todos os resultados 
foram considerados significativos para uma probabilidade de significância inferior a $5 \%(\mathrm{p}<0,05)$, tendo, portanto, pelo menos $95 \%$ de confiança nas conclusões apresentadas.

As bases de dados consultadas neste trabalho foram a Scientific Electronic Library Online (SciELO), Pubmed Central (PMC), Literatura Latino-americana e do Caribe em Ciências da Saúde (LILACS), Medical Literature Analysis and Retrievel System Online (MEDLINE), além de instituições referência, como o Instituto Nacional de Câncer e a American Cancer Society.

\section{Resultados}

Foram analisados todos os 398 questionários aplicados. Dentre as participantes da pesquisa, $62,8 \%$ estavam na faixa etária dos 50 a 69 anos, com média de idade de 52,2 \pm 9,5 anos. Em relação a outras características sociodemográficas, 52,8\% estavam casadas ou em união estável, 50,4\% apresentavam apenas o ensino fundamental parcial ou completo e 38,2\% possuíam ensino médio completo. A incidência de CA de mama encontrada foi de 2,5\% das participantes A média do índice de massa corporal calculada foi de $28,1 \pm 5,5$, sendo que a maioria apresentava peso normal, sobrepeso e obesidade grau I, como mostrado na Tabela 1.

Tabela 1. Características sociodemográficas das mulheres atendidas no Centro de Ensino e Atenção à Saúde da Comunidade, em Belo Horizonte, Minas Gerais, Brasil, 2019.

\begin{tabular}{|c|c|c|}
\hline Característica Observada & \multicolumn{2}{|c|}{ Frequência } \\
\hline & $\bar{N}$ & $\%$ \\
\hline \multicolumn{3}{|l|}{ Idade - em anos (n: 398) } \\
\hline Média \pm DP & \multicolumn{2}{|c|}{$52,2 \pm 9,5$} \\
\hline I.C. da média (95\%) & \multicolumn{2}{|c|}{$(51,2 ; 53,1)$} \\
\hline Mediana $\left(\mathrm{Q}_{1}-\mathrm{Q}_{3}\right)$ & \multicolumn{2}{|c|}{$53,0(45,0-60,0)$} \\
\hline Mínimo - Máximo & \multicolumn{2}{|c|}{$28,0-69,0$} \\
\hline Classificação de IMC* (n: 322) & & \\
\hline Abaixo do peso $\left(<18 \mathrm{~kg} / \mathrm{m}^{2}\right)$ & 6 & 1,8 \\
\hline Peso Normal $\left(\geq 18 \mathrm{e} \leq 25 \mathrm{~kg} / \mathrm{m}^{2}\right)$ & 102 & 31,7 \\
\hline Sobrepeso $\left(>25 \mathrm{e} \leq 30 \mathrm{~kg} / \mathrm{m}^{2}\right)$ & 102 & 31,7 \\
\hline Obesidade grau I $\left(>30\right.$ e $\left.\leq 35 \mathrm{~kg} / \mathrm{m}^{2}\right)$ & 76 & 23,6 \\
\hline Obesidade grau II $\left(>35 \mathrm{e} \leq 40 \mathrm{~kg} / \mathrm{m}^{2}\right)$ & 29 & 9,0 \\
\hline Obesidade grau III $\left(\geq 40 \mathrm{~kg} / \mathrm{m}^{2}\right)$ & 7 & 2,2 \\
\hline \multicolumn{3}{|l|}{ Escolaridade (n: 385) } \\
\hline Fundamental incompleto & 122 & 31,7 \\
\hline Fundamental completo & 72 & 18,7 \\
\hline Ensino Médio completo & 147 & 38,2 \\
\hline Superior incompleto & 18 & 4,7 \\
\hline Superior completo & 22 & 5,7 \\
\hline Pós-graduação & 4 & 1,0 \\
\hline
\end{tabular}

n: valor absoluto da amostra; DP: desvio-padrão; IC: intervalo de confiança; Fonte: Autores.

Quando questionadas sobre as fontes de informação acerca da doença, 97,2\% (n: 387) das mulheres responderam à questão. A mídia (jornais, revistas e televisão) correspondeu a fonte de informação sobre a doença para 60,50\% da amostra, seguida pela Unidade Básica de Saúde. A Campanha Outubro Rosa foi citada, porém a maioria das participantes nunca tiveram acesso às atividades promovidas por essa campanha (Gráfico 1). 
Gráfico 1. Principais fontes de informação sobre câncer de mama citadas pelas mulheres atendidas no Centro de Ensino e Atenção à Saúde da Comunidade, em Belo Horizonte, Minas Gerais, Brasil, 2019.

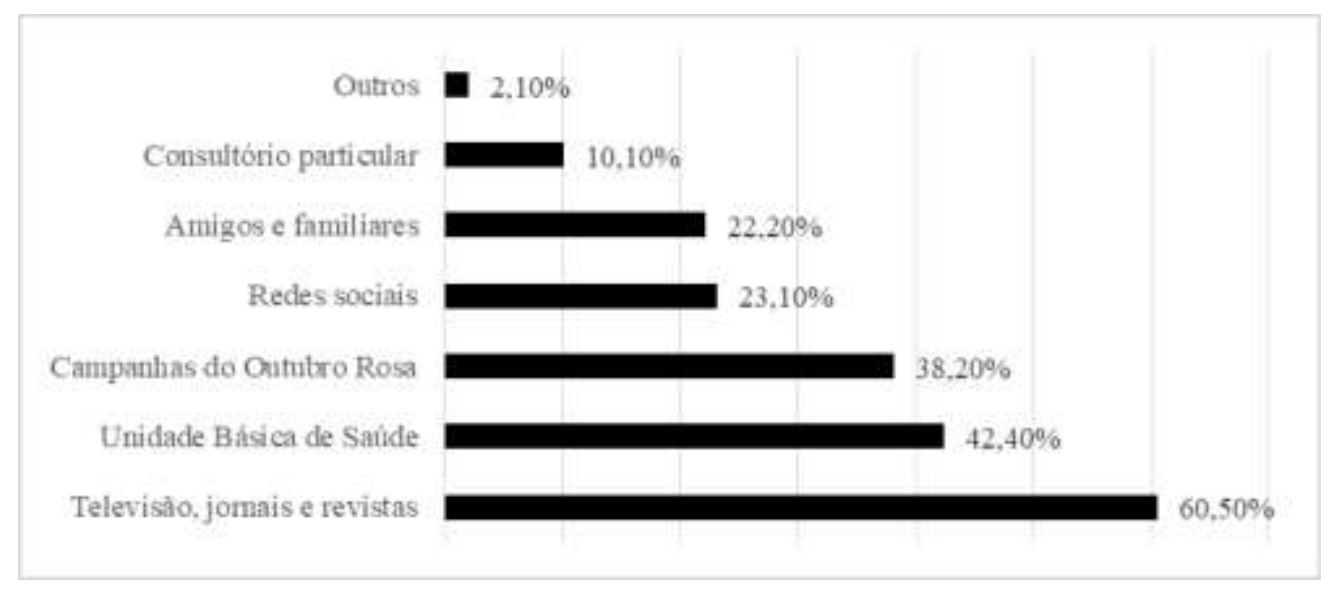

Fonte: Autores.

Gráfico 2: Condições reconhecidas como fatores de risco para neoplasia da mama pelas mulheres atendidas no Centro de Ensino e Atenção à Saúde da Comunidade, em Belo Horizonte, Minas Gerais, Brasil, 2019.

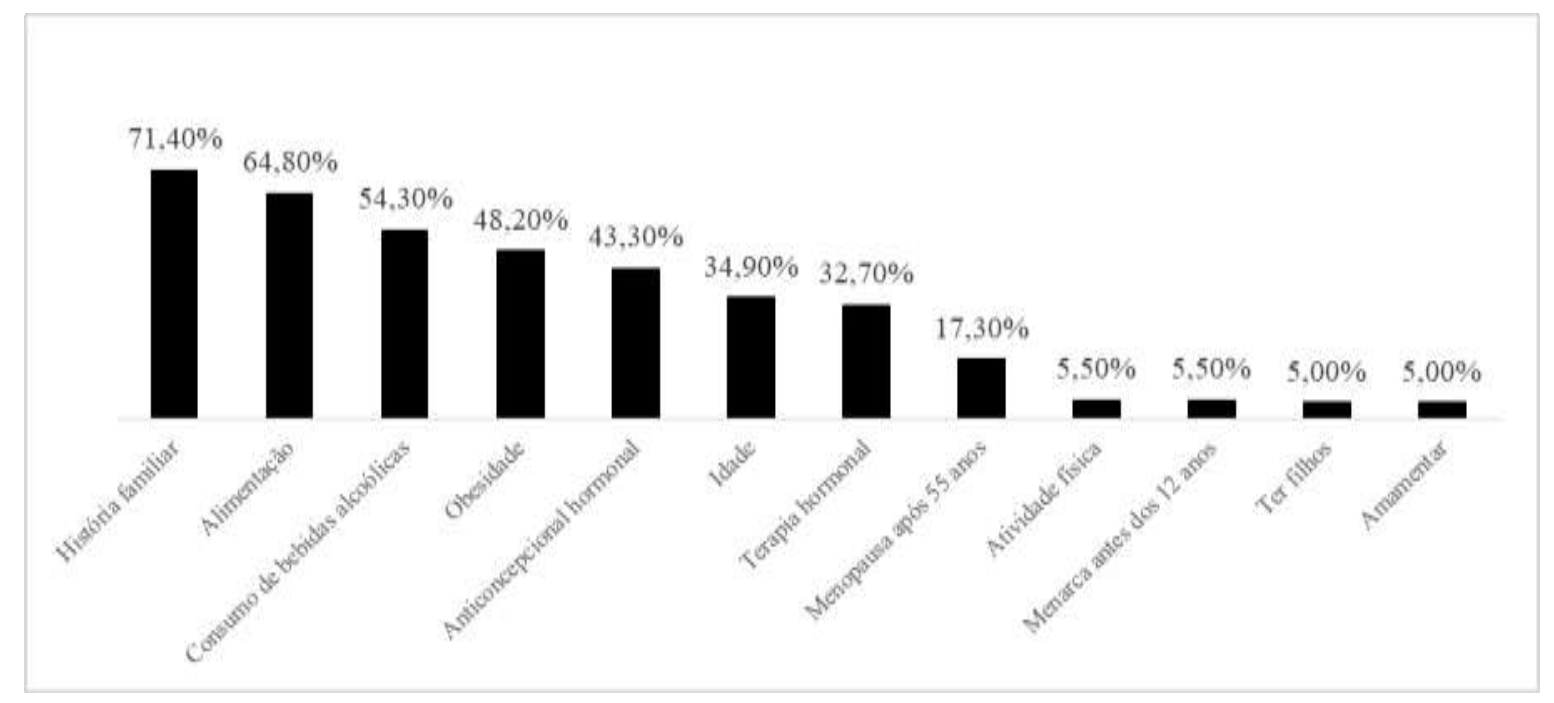

Fonte: Autores.

Dentre os 12 itens listados para serem assinalados como possíveis fatores de risco para o câncer de mama (Gráfico 2), a média de acertos foi de apenas 1,38 (DP \pm 1,74). Das 398 mulheres, 39 conheciam apenas um fator de risco, 38 dois, 45 três, 41 quatro, 21 cinco e cinco conheciam seis fatores. Dentre os sete fatores de risco modificáveis (alimentação, consumo de bebidas alcoólicas, obesidade, terapia hormonal, atividade física, amamentar e ter filhos) a média de acertos foi de 1,76 (DP \pm $1,33)$.

Na opinião de 83 mulheres, a genética é a principal causa do CA de mama, sendo que 192 responderam à questão. Além disso, ter um parente de primeiro grau com CA de mama foi a opção mais assinalada como fator de risco. As mulheres com nível de ensino superior tiveram 11,08 vezes (OR: 11,08; IC: 2,60 - 47,19; p: 0,001) mais chance de marcar essa opção. Isso também foi observado com as participantes que tinham faixa etária menor que 50 anos, que apresentaram 2,1 vezes (OR: 2,1; IC: 1,3 - 3,4; p: 0,002) mais chance. A segunda opção mais marcada como fator de risco para a neoplasia da mama foi a alimentação. 
Em relação a outras comparações sobre os fatores de risco, mulheres com idade entre 50 e 69 anos apresentaram maior chance de considerarem o consumo de bebidas alcoólicas (OR: 1,85; IC: 2,80 - 1,24; p: 0,003), terapia hormonal (OR: 1,96; IC: 3,13 - 1,25; p: 0,003) e o uso de anticoncepcional hormonal (OR: 1,7; IC: 2,57 - 1,1; p: 0,016) como fatores de risco para a doença, quando comparados àquelas com menos de 50 anos (Tabela 2).

Ademais, as participantes com nível de escolaridade fundamental têm 3,45 vezes (OR inversa: 1/0,26 =3,85; IC: 1,28 - 13,94; p: 0,029) mais chance de dizer que a "menopausa após os 55 anos" aumenta o risco de neoplasia mamária, do que aquelas com nível de escolaridade superior. As mulheres obesas ou com sobrepeso apresentaram 1,7 vezes (IC: 1,1 - 2,7; p: 0,022 ) mais chance de considerar a obesidade como um fator de risco do que mulheres com IMC normal ou baixo (Tabela 2).

Tabela 2. Distribuição da amostra de acordo com a identificação dos fatores de risco para câncer de mama, segundo análise de significância estatística dos dados encontrados.

\begin{tabular}{|c|c|c|c|c|c|}
\hline Característica & $\begin{array}{l}\text { História } \\
\text { familiar }\end{array}$ & $\begin{array}{c}\text { Consumo de } \\
\text { bebida alcoólica }\end{array}$ & $\begin{array}{c}\text { Terapia } \\
\text { hormonal }\end{array}$ & $\begin{array}{c}\text { Anticoncepcional } \\
\text { hormonal }\end{array}$ & Obesidade \\
\hline Idade $<50$ anos & $\begin{array}{c}\text { OR: } 2,1 \\
\text { IC: } 1,3-3,4 \\
\text { p: } 0,002\end{array}$ & $\overline{\mathrm{NS}}$ & $\overline{\mathrm{NS}}$ & $\overline{\mathrm{NS}}$ & $\overline{\mathrm{NS}}$ \\
\hline Idade $\geq 50$ e $\leq 69$ & NS & $\begin{array}{c}\text { OR: } 1,85 \\
\text { IC: } 1,24-2,8 \\
\text { p: } 0,003\end{array}$ & $\begin{array}{c}\text { OR: } 1,96 \\
1,25-3,13 \\
0,003\end{array}$ & $\begin{array}{c}\text { OR: } 1,7 \\
\text { IC: } 1,1-2,57 \\
\text { p: } 0,016\end{array}$ & NS \\
\hline Ensino superior & $\begin{array}{c}\text { OR: } 11,08 \\
\text { IC: } 2,6-47,19 \\
\text { p: } 0,001\end{array}$ & NS & NS & NS & NS \\
\hline $\begin{array}{l}\text { Obesidade ou } \\
\text { sobrepeso }\end{array}$ & NS & NS & NS & NS & $\begin{array}{c}\text { OR: } 1,7 \\
\text { IC: } 1,1-2,7 \\
\text { p: } 0,022\end{array}$ \\
\hline
\end{tabular}

NS: Não significante; OR: Odds ratio; IC: Intervalo de confiança de 95\%; p: probabilidade de significância. Fonte: Autores.

Já ter realizado a mamografia não influenciou no número de opções corretas assinaladas. A média de acertos das mulheres que realizaram o exame foi de 1,4 ( $\mathrm{DP} \pm 1,8$ ) e a média das que não realizaram foi de 1,5 ( $\mathrm{DP} \pm 1,6)$. Ademais, ter participado de campanhas do Outubro Rosa também não representou diferença estatística significativa entre o total de acertos.

\section{Discussão}

O presente estudo identificou que o conhecimento das participantes sobre a prevenção primária do câncer de mama é limitado. Avaliando as características socioeconômicas encontradas, observa-se que a faixa etária predominante foi de mulheres com idade superior a 50 anos. A maioria das participantes relataram união estável e Derenzo, Costa, Costa, Francisqueti \& Bernucci (2017) apontam que a relação entre o conhecimento de fatores de risco para o câncer de mama e o estado civil é incerta, diferente do que ocorre quando a influência de outros possíveis determinantes sociais - renda, tipo de ocupação e nível de escolaridade - são considerados (Freitas \& Weller, 2019).

Neste estudo, foi observado que as mulheres com sobrepeso e obesidade eram maioria e que, curiosamente, estas tinham maior chance de considerar que a obesidade aumenta o risco de desenvolver a neoplasia mamária. A obesidade é fator de risco para o CA câncer de mama na pós-menopausa, além de ser associada à pior resposta terapêutica e à maiores taxas de recorrência (Batiston et al, 2011). O mecanismo pelo qual ocorre tal associação ainda não é totalmente elucidado, mas algumas hipóteses incluem a secreção irregular de estrogênio e aumento de marcadores inflamatórios, característicos da obesidade. 
Outras condições, como a alteração de adipocinas, da insulina e da microbiota intestinal são alvos atuais de estudos (Nogueira et al, 2020). Ademais, a obesidade e sobrepeso relacionam-se com condições prejudiciais, tais como doenças cardiovasculares, diabetes e sedentarismo (ACS, 2020).

Quando avaliadas as principais fontes de informação sobre a doença, foi constatado um predomínio da mídia, seguida da UBS. Estudos similares, realizados em Paranavaí-PR (Derenzo et al, 2017) e na cidade de São Paulo (Santos \& Chubaci, 2011), também apontaram os meios de comunicação como a principal fonte de informação das mulheres participantes. A mídia televisiva sobressaiu em todos os estudos, o que já era esperado, tendo em vista seu inquestionável papel como meio de comunicação de massa e como mídia de mais fácil acesso para a população brasileira (Instituto Brasileiro de Geografia e Estatística [IBGE], 2017). A mídia, portanto, exerce papel essencial no combate ao CA de mama no Brasil. A divulgação científica sobre os diversos tipos de CA através das mídias impressa e digital, foi investigada em um estudo que analisou 195 reportagens de 54 jornais das diversas regiões do país. O tema mais prevalente foi a prevenção de neoplasias, abordada em $40 \%$ das reportagens. Infelizmente, 10\% das publicações da região Sudeste apresentavam informações equivocadas (Jurberg, Gouveia \& Belisário, 2006).

Em outros estudos realizados em Viçosa-MG (Martins, Barbosa \& Cezar, 2014) e Dourados-MS (Batiston, Tamaki, Souza \& Santos, 2011), a maioria das participantes relatou que a informação acerca do CA de mama foi prestada por um profissional de saúde, o que não foi observado neste estudo. Esse dado mostra a necessidade de tornar as ações de saúde pública mais efetivas na conscientização e sensibilização das mulheres e dos profissionais de saúde em relação à neoplasia mamária, em Belo Horizonte. O fortalecimento do vínculo entre profissionais de saúde e pacientes possibilitaria a disseminação de conhecimento consistente e preciso acerca das características não apenas das doenças da mama, mas também de outras condições de saúde que afetam a população feminina. A aproximação da fonte de informação com o público-alvo poderia estimular a aderência às ações propostas.

As atividades de educação em saúde são estratégias fundamentais para consolidar o conhecimento sobre as doenças, contribuindo com a prevenção primária. No contexto das neoplasias mamárias, ao avaliar as campanhas do Outubro Rosa, observamos que estas foram citadas como fonte de informação por 38,2\% das mulheres. No entanto, a maioria das participantes $(79,6 \%)$ relatou que nunca participou de atividades promovidas durante essa campanha e já ter participado não influenciou estatisticamente o número de acertos acerca dos fatores de risco para a doença. Na pesquisa supracitada, realizada em Viçosa, $73 \%$ das mulheres afirmaram que já ouviram sobre o Outubro Rosa, mas quando avaliado como fonte de informação para estas, apenas $0,8 \%$ o citaram (Martins et al, 2014).

Em contrapartida, Quintanilha et al. demonstraram em 2019, a partir de dados coletados pelo googletrends, que há um aumento nas pesquisas na internet por CA de mama durante o mês de outubro. Esse fato demonstra a capacidade que as campanhas possuem de despertar o interesse da população sobre um tema, mas nota-se que no caso do Outubro Rosa isso tem contribuído insuficientemente para a consolidação do conhecimento de boa parte de seu público-alvo. Por outro lado, os achados também reforçam o relevante papel das mídias digitais como meio de adquirir conhecimento.

O conhecimento de fatores de risco para o CA de mama foi mensurado através da média de acertos dos fatores listados no questionário. Apenas 47,5\% das participantes acertaram pelo menos um dos fatores de risco, sem apontar também algum fator protetor. A alimentação, o consumo de bebidas alcoólicas e a obesidade foram as principais condições modificáveis reconhecidas como de risco para a doença. Resultados semelhantes foram encontrados por Batiston et al. (2011), estudo no qual a maioria das condições reconhecidas como de risco era do tipo potencialmente modificável. Não se pode afirmar que o conhecimento sobre os fatores de risco influencia diretamente a mudança de comportamento da população feminina, como aponta o próprio autor no texto. No entanto, é essencial fortalecer a ideia de que muitos fatores podem e devem ser modificados, sendo necessária uma avaliação e consequente mudança no estilo de vida da sociedade atual. 
Diversos estudos destacam a importância do controle do peso corporal, prática de atividade física, adesão a uma dieta saudável e redução do consumo de bebidas alcoólicas (Coughlin \& Smith, 2015). Recentemente, a American Cancer Society (ACS) atualizou as recomendações para dieta e exercícios físicos, mostrando que a atividade física regular de moderada e alta intensidades é capaz de diminuir o risco de CA de mama tanto na pré-menopausa, quanto na pós-menopausa. Por outro lado, o consumo de bebidas alcoólicas aumenta o risco nessas duas fases da vida da mulher. A ACS também indica a alimentação baseada em produtos naturais e pobre em alimentos industrializados (ACS, 2020). É importante notar que essas ações trariam não apenas a diminuição na incidência do CA de mama e de sua morbimortalidade, mas também a evolução na promoção da saúde da mulher integralmente.

A associação entre o desenvolvimento da neoplasia mamária e fatores genéticos e hereditários é frequentemente estabelecida. No nosso estudo não foi diferente, a maioria das participantes consideraram o fator genético a principal causa do CA de mama. Além disso, a história familiar (parentes de primeiro grau) foi opção mais assinalada como fator de risco para a doença. Esses achados são compatíveis com o estudo de Derenzo et al. (2017) e de Freitas e Weller (2019), em que a história familiar foi citada por, respectivamente, $52,17 \%$ e $85,6 \%$ das entrevistadas, sendo o fator de risco mais apontado. De fato, tanto o risco de desenvolver a neoplasia, quanto o risco de recorrência estão aumentados nos casos de histórico de parente de primeiro grau acometido pela doença. Porém, é importante notar que somente $10 \%$ dos casos ocorrem devido a fatores hereditários, como as mutações germinativas nos genes BRCA1 e BRCA2 (Instituto Nacional de Câncer José Alencar Gomes da Silva [INCA], 2020).

Outro ponto importante diz respeito aos fatores endócrinos e da história reprodutiva da mulher, muito associados ao estímulo estrogênico endógeno ou exógeno. A menarca precoce, menopausa tardia, primiparidade após os 30 anos ou nuliparidade e a terapia hormonal pós-menopausa são fatores de risco para o CA de mama (World cancer research fund [WCRF], 2017). As participantes reconheceram principalmente o uso de contraceptivos hormonais como condição predisponente, fator inconsistente na literatura (Pinho \& Coutinho, 2005) e que no nosso estudo não foi considerado como um fator de risco. Os anticoncepcionais hormonais combinados podem causar discreto aumento do risco de neoplasia em jovens, caso o uso seja atual ou de início recente (WCRF, 2017). Ademais, "ter filhos" e "amamentar" são importantes fatores de proteção e foram apontados como fatores de risco para a doença por 10\% das mulheres.

O presente trabalho tem como limitação que sua população foi restrita a região sudeste do Brasil e a pacientes que frequentam o serviço público de saúde, portanto seus dados não podem ser extrapolados para outras regiões e para mulheres que frequentam a rede privada. Por ser um estudo transversal também não é possível inferir de que modo o conhecimento das mulheres sobre os fatores de risco para a neoplasia de mama pode interferir na sua mudança de comportamento e prevenção da doença.

\section{Conclusão}

O conhecimento limitado acerca da prevenção primária do CA de mama, encontrado neste estudo, corrobora os dados trazidos por estudos brasileiros anteriores. Isso demonstra que a alta morbimortalidade associada às neoplasias da mama não tem sido acompanhada de respostas eficazes de combate dessa doença e de promoção da saúde da mulher. A importante atuação das mídias e dos profissionais de saúde na disseminação de conhecimento, demonstra que é necessário ampliar as discussões, com divulgação de dados seguros e fortalecimento do vínculo entre promotores de saúde e população. Os locais de atendimentos, como as UBS e os ambulatórios, deveriam aproveitar melhor os períodos de espera, de realização de exames e de consulta, em que há maior proximidade com as mulheres. As campanhas do outubro rosa precisam ser mais inclusivas, interativas e melhor compreendidas pelas participantes. Ademais, não basta que a informação alcance o público-alvo, é 
essencial que as próprias mulheres saibam da sua responsabilidade individual na prevenção das neoplasias da mama e atuem ativamente na busca de uma melhor qualidade de vida.

\section{Referências}

American Cancer Society (2020). Guideline for Diet and Physical Activity for Cancer Prevention. CA Cancer J Clin, 0, 1-27.

Batiston, A. P., Tamaki, E. M., Souza, L. A. \& Santos, M. L. M. (2011). Conhecimento e prática sobre os fatores de risco para o câncer de mama entre mulheres de 40 a 69 anos. Rev. Bras. Saúde Matern. Infant. Recife, 11 (2), 163-171. 10.1590/S1519-38292011000200007

Coughlin, S. S. \& Smith, S. A (2015). The Impact of the Natural, Social, Built, and Policy Environments on Breast Cancer. J Environ Health Sci., 1(3). $10.15436 / 2378-6841.15 .020$

Derenzo, N., Costa, M. A. R., Costa, C. K. F., Francisqueti, V. \& Bernucci, M. P (2017). Conhecimento de mulheres sobre fatores relacionados ao câncer de mama. Rev Enferm UFSM, 7(3), 436-447. 10.5902/2179769225641

Freitas, Â.G. Q., \& Weller, M, 2019. Women's knowledge about risk factors of breast cancer in a Brazilian community. Women Health, 59(5), 558-568.10.1080/03630242.2018.1516266

Harvie, M., \& Howell, A.,Evans, G (2015).Can Diet and Lifestyle Prevent Breast Cancer: What Is the Evidence?. ASCO Educational Book, 66-73. $\rightarrow$ (Harvie, Howell \& Evans, 2015).10.14694/EdBook_AM.2015.35.e66.

Howell, A., Anderson, A. S., Clarke, R.B., Duffy, S. W., Evans, D. G., Garcia-Closas, M., Gescher, A. J., Key, T. J., Saxton, J. M., \& Harvie, M. N (2014). . Risk determination and prevention of breast cancer. Breast Cancer Res, 16(5), 446. 10.1186/s13058-014-0446-2

Instituto Nacional de Câncer José Alencar Gomes da Silva (2019). Estimativa 2020: incidência de câncer no Brasil. 120p.

Instituto Nacional de Câncer José Alencar Gomes da Silva (2020). Câncer de mama: prevenção e fatores de risco. <https://www.inca.gov.br/tipos-decancer/cancer-de-mama/profissional-de-saude>.

Instituto Brasileiro de Geografia e Estatística (2017). Pesquisa nacional por amostras de domicílio contínuas: Acesso à Internet e à televisão e posse de telefone móvel celular para uso pessoal. <https://biblioteca.ibge.gov.br/visualizacao/livros/liv101631_informativo.pdf〉.

Inumaru, L. E., Silveira, E. A., \& Naves, M. M. V (2009). Fatores de risco e de proteção para câncer de mama: uma revisão sistemática. Cadernos de Saúde Pública. 27(7), 1259-1270.10.1590/S0102-311X2011000700002

Jeronimo, A. F. A., Freitas, A. G. Q. \& Weller, M. (2011). Fatores de risco do câncer de mama e o conhecimento sobre a doença: revisão integrativa de estudos Latino Americanos. Ciência \& Saúde Coletiva, 22(1), 135-149. 10.1590/1413-81232017221.09272015

Jurberg, C , Gouveia, M. .E. \& Belisário, C (2006). Na mira do câncer: o papel da mídia brasileira. Revista Brasileira de Cancerologia, 52 (2), $139-146$.

Kolak, A., Kamińska, M., Sygit, K., Budny, A., Surdyka, D., Kukiełka-Budny, B. \& Burdan, F. (2017). Primary and secondary prevention of breast cancer. Ann Agric Environ Med, 24(4), 549-553. 10.26444/aaem/75943.

Martins, A. F. H., Barbosa, T. R. C. G. \& Cezar, C. L (2014). Análise da campanha do Outubro Rosa de Prevenção do câncer de mama em Viçosa MG. Universidade Federal de Viçosa, 14(2), 539-556. URI: http://www.locus.ufv.br/handle/123456789/12978

Nogueira, T. R., Araujo, C. G. B, Cldas, D. R. C., Maciel, E. M., Silva, M. C. M. \& Rodrigues, G. P. (2020). Obesidade e Câncer de mama: Algumas evidências científicas e vias de interação. Research, Society and Development, 9 (4). http://dx.doi.org/10.33448/rsd-v9i4.2675 1

Pinho, V. F. S. \& Coutinho, E. S. F (2005). Risk factors for breast cancer: a systematic review of studies with female samples among the general population in Brazil. Cad. Saúde Pública, Rio de Janeiro, 21(2), 351-360. 10.1590/S0102-311X2005000200002

Quintanilha, L. F., Souza L. N., Sanches, D., Demarco, R. S. \& Fukutani, F. K. (2019). The impact of cancer campaigns in Brazil: a Google Trends analysis. Ecancer, 13, 963. 10.3332/ecancer.2019.963

Rodrigues, J. D., Cruz, M. S., \& Paixão, A. N (2015). Uma análise da prevenção do câncer de mama no Brasil. Ciência \& Saúde Coletiva, 20(10). 31633176.10.1590/1413-812320152010.20822014

Santos, G. D. \& Chubaci, R. Y. S (2011). O conhecimento sobre o câncer de mama e a mamografia das mulheres idosas frequentadoras de centros de convivência em São Paulo. Ciência \& Saúde Coletiva, 16(5), 2533-2540.https://doi.org/10.1590/S1413-81232011000500023

Santos M. O. (2018). Estimativa 2018: Incidência de Câncer no Brasil. Revista Brasileira de Cancerologia, 64(1), 119-120

Silva, A. S. et al (2020). Análise da qualidade de vida em mulheres após o diagnóstico de câncer de mama em hospital de referência oncológica no Nordeste brasileiro. Research, Society and Development, 9 (11). http://dx.doi.org/10.33448/rsd-v9i11.10218

World cancer research fund, American institute for cancer research (2017). Breast cancer report. <https://www.wcrf.org/sites/default/files/Breast-cancerreport.pdf>.

World Health Organization (2020). Câncer de mama: prevenção e controle. <http://www.who.int/cancer/detection/breastcancer/en/>. 